\title{
uPAR as a Glioma Imaging Target
}

\author{
Kenji Hirata and Nagara Tamaki \\ Department of Nuclear Medicine, Graduate School of Medicine, Hokkaido University, Sapporo, Japan
}

G liomas account for $70 \%$ of primary brain tumors (1). Among the various types of glioma, glioblastoma is the most aggressive astrocytic tumor, being classified grade IV by the World Health Organization (2). Although CT and MRI are indispensable in providing morphologic information, functional imaging using PET plays an important role in grading tumors, delineating tumor boundaries, monitoring treatment, and discriminating recurrent tumor from treatment-induced changes (3). ${ }^{18} \mathrm{~F}$-FDG is the best-established PET tracer for various malignancies; however, the high glucose metabolism of the brain prevents accurate evaluation of brain neoplasms using ${ }^{18}$ F-FDG PET. In addition, although higher-grade gliomas metabolize more glucose than lower-grade gliomas, even glioblastomas sometimes show lower uptake than the surrounding brain tissue, making ${ }^{18}$ F-FDG PET images difficult to interpret-especially in evaluating tumor expansion. In this context, other tracers for brain tumors have been extensively investigated over the past few decades. Among them, amino acid tracers such as ${ }^{11} \mathrm{C}$-methionine $(4,5)$ and ${ }^{18}$ F-fluoroethyltyrosine $(6,7)$ have been the most successful, followed by hypoxia imaging agents such as ${ }^{18} \mathrm{~F}$-fluoromisonidazole $(8)$ and nucleic acid analogs such as ${ }^{18} \mathrm{~F}$-fluorothymidine (9). Now, urokinasetype plasminogen activator receptor (UPAR) has been added to the array of available tracers for imaging.

\section{See page 272}

UPAR is a glycosylphosphatidylinositol-anchored receptor that is located on the cell surface and binds the serine protease urokinasetype plasminogen activator (10). UPAR is important in regulating extracellular matrix proteolysis, cell-extracellular matrix interactions, and cell signaling and has limited expression under normal conditions. Some exceptions are keratinocytes during wound healing and brain tissue that has undergone ischemic or traumatic changes (11). Cancer cells also express uPAR. They make use of uPAR because proteolytic degradation of the extracellular matrix is essential for tumor invasion and metastasis. The intensity of UPAR expression is associated with poor prognosis in many malignancies, as demonstrated by nearly 100 papers published between 1990 and 2010 describing UPAR expression in cancers of the bladder, breast, colon and

Received Sep. 19, 2015; revision accepted Sep. 22, 2015.

For correspondence or reprints contact: Kenji Hirata, Department of Nuclear Medicine, Graduate School of Medicine, Hokkaido University, Kita 15, Nishi 7, Kita-Ku, Sapporo, Hokkaido, Japan 060-8638.

E-mail: khirata@med.hokudai.ac.jp

Published online Oct. 1, 2015.

COPYRIGHT (c) 2016 by the Society of Nuclear Medicine and Molecular Imaging, Inc.

DOI: $10.2967 /$ jnumed.115.166231 rectum, stomach, blood, liver, lung, pancreas, and prostate (10). Glioblastomas also exhibit increased UPAR expression, with a greater level of expression indicating higher invasiveness and shorter survival. Such characteristics raised the possibility of using uPAR as an imaging agent for glioblastoma.

Ploug's group contributed greatly to the development of uPAR imaging agents. They developed several peptide-derived antagonists of UPAR and described them in a 2001 article (12). Interestingly, according to that article, these compounds were initially designed for chemotherapy rather than for radionuclide therapy, and some of the data indicated the inhibitory effect of peptide-derived antagonists of uPAR on tumor invasion (12). Based on AE105, which is one of the antagonist products proposed by Ploug's group, Li et al. (also Ploug's colleagues) first developed a radioactive uPAR ligand, ${ }^{64} \mathrm{Cu}$-DOTA-AE105 (13). More recently, the Kjaer group performed a series of important steps to modify and characterize radioactive uPAR ligands. They developed and examined the radioactive uPAR ligands ${ }^{68} \mathrm{Ga}$-DOTA-AE105- $\mathrm{NH}_{2}(14),{ }^{68} \mathrm{Ga}-\mathrm{NODAGA}-\mathrm{AE} 105-\mathrm{NH}_{2}$ (14), ${ }^{18}$ F-AlF-NOTA-AE105 (15), ${ }^{64} \mathrm{Cu}$-CB-TE2A-PA-AE105 (16), and ${ }^{64} \mathrm{Cu}$-DOTA-AE105 (17). In this issue of The Journal of Nuclear Medicine, Persson et al. from the Kjaer group present evidence of the usefulness of two new uPAR PET tracers, ${ }^{64} \mathrm{Cu}-\mathrm{NOTA}-\mathrm{AE} 105$ and ${ }^{68} \mathrm{Ga}-\mathrm{NOTA}-\mathrm{AE} 105$, in glioblastoma imaging (18). Three highlights of their study are, first, that an orthotopic glioblastoma model was used to demonstrate strong accumulation of both tracers in the tumor; second, that compared with ${ }^{18} \mathrm{~F}$-fluoroethyltyrosine, the new tracers showed lower absolute uptake values but higher tumor-to-background ratios; and third, that autoradiography revealed the intratumoral tracer distribution, which resembled the immunohistochemical staining of UPAR.

In previous investigations by Persson et al., a tumor was implanted in subcutaneous tissue or muscle because that approach is relatively easy and less time-consuming. However, there are always criticisms against such an approach from the viewpoint of the limited similarity between spontaneous cancers and implanted cell lines. In the present study, Persson et al. established cell cultures sampled from a glioblastoma patient and injected the tumor cells into the brain tissue of nude mice. This method is technically demanding but worth establishing. Although this model is still somewhat different from naturally occurring tumors, we consider the present findings to provide more reliable evidence justifying clinical studies of the new tracers. Attention should be paid to the radionuclides used by Persson et al. Whereas the production of ${ }^{18} \mathrm{~F},{ }^{11} \mathrm{C}$, and ${ }^{64} \mathrm{Cu}$ (half-life, $12.7 \mathrm{~h}$ ) requires a cyclotron, ${ }^{68} \mathrm{Ga}$ (half-life, $68 \mathrm{~min}$ ) is produced by a ${ }^{68} \mathrm{Ge} /{ }^{68} \mathrm{Ga}$ generator, and thus the preparation of ${ }^{68} \mathrm{Ga}$-DOTA-AE105 does not require an in-house cyclotron. Conversely, the relatively short half-life of ${ }^{68} \mathrm{Ga}$ may restrict imaging at a late phase. It is important to understand the advantages and disadvantages of different radionuclides when considering them for imaging. In any event, it is good news that both ${ }^{64} \mathrm{Cu}$ and ${ }^{68} \mathrm{Ga}$ can be used to label $\mathrm{AE} 105$ peptide. 
A few issues are not addressed by Persson et al. in their new report. First, as they admit in the Discussion section, their uPAR ligands have strong species specificity; the ligands bind with 200-fold higher affinity to human uPAR than to mouse uPAR (19). This may be the significant factor causing the high tumor-to-background ratio in the study (i.e., the tumor is of human origin but the background is of mouse origin). uPAR ligands are also different from analogs of more ubiquitous substrates such as glucose (e.g., $\left.{ }^{18} \mathrm{~F}-\mathrm{FDG}\right)$ and amino acids (e.g., ${ }^{11} \mathrm{C}$-methionine and ${ }^{18} \mathrm{~F}$-fluoroethyltyrosine). Thus, clinical studies using uPAR ligands are expected to result in lower contrast than in the present study. Second, although the autoradiography images were similar to the immunostaining of uPAR, the accumulation was not compared quantitatively. The quantity in the autoradiography image (Fig. 6C) cannot be determined because the entire positive area is shown in red. The question of whether the intensity of tracer uptake reflects the intensity of UPAR expression remains unanswered. This question is particularly important because in clinical settings the tracers are expected to be used to estimate UPAR expression for risk stratification. This study used just one cell line obtained from a single patient. Different glioblastomas expressing different levels of UPAR must be examined in order to test the quantitative performance of the tracers. Third, together with previous papers, the new study by Persson et al. presents several uPAR tracer candidates. Comparative studies need to determine the most feasible candidate before clinical studies can take place, and the Kjaer group recently took the first of these UPAR tracers into a human trial (20).

We also hope that future studies will determine whether information obtained from uPAR imaging is an independent factor in determining patient prognosis. After several pilot studies are performed, it will be necessary to conduct studies of large populations with multivariate analyses that include known prognosis factors such as age, glioma grade, and surgical procedure. Comparisons with established tracers such as ${ }^{18} \mathrm{~F}$-FDG, ${ }^{11} \mathrm{C}$-methionine, ${ }^{18} \mathrm{~F}$-fluoroethyltyrosine, and ${ }^{18} \mathrm{~F}$-fluoromisonidazole are also important. The question of whether the imaging technique can be used for monitoring treatment response is also of interest. The ultimate goal of tumor imaging is, of course, to improve patient outcomes.

Before closing, we would like to mention a uPAR-targeted radionuclide therapeutic agent, ${ }^{177} \mathrm{Lu}$-DOTA-AE105, which has been tested by Persson et al. using colorectal cancer xenografts (21). This therapy was shown to reduce both tumor size and the rate of uPARpositive cells without producing significant side effects in the kidneys and other organs. It is greatly beneficial that the same compound can be used for both imaging and therapy, because the imaging technique directly predicts the treatment effects. Preclinical and clinical studies further investigating these agents are eagerly awaited.

\section{DISCLOSURE}

No potential conflict of interest relevant to this article was reported.

\section{REFERENCES}

1. Ohgaki H. Epidemiology of brain tumors. Methods Mol Biol. 2009;472:323-342.

2. Louis DN, Ohgaki H, Wiestler OD, et al. The 2007 WHO classification of tumours of the central nervous system. Acta Neuropathol (Berl). 2007;114: 97-109.

3. Heiss WD, Raab P, Lanfermann H. Multimodality assessment of brain tumors and tumor recurrence. J Nucl Med. 2011;52:1585-1600.

4. Herholz K, Holzer T, Bauer B, et al. ${ }^{11} \mathrm{C}$-methionine PET for differential diagnosis of low-grade gliomas. Neurology. 1998;50:1316-1322.

5. Kobayashi K, Hirata K, Yamaguchi S, et al. Prognostic value of volume-based measurements on ${ }^{11} \mathrm{C}$-methionine PET in glioma patients. Eur J Nucl Med Mol Imaging. 2015;42:1071-1080.

6. Wester HJ, Herz M, Weber W, et al. Synthesis and radiopharmacology of O-(2$\left[{ }^{18} \mathrm{~F}\right]$ fluoroethyl)-L-tyrosine for tumor imaging. J Nucl Med. 1999;40:205-212.

7. Jansen NL, Suchorska B, Wenter V, et al. Prognostic significance of dynamic ${ }^{18} \mathrm{~F}-\mathrm{FET}$ PET in newly diagnosed astrocytic high-grade glioma. J Nucl Med. 2015;56:9-15.

8. Hirata K, Terasaka S, Shiga T, et al. ${ }^{18} \mathrm{~F}$-fluoromisonidazole positron emission tomography may differentiate glioblastoma multiforme from less malignant gliomas. Eur J Nucl Med Mol Imaging. 2012;39:760-770.

9. Muzi M, Spence AM, O'Sullivan F, et al. Kinetic analysis of $3^{\prime}$-deoxy- $3^{\prime}-{ }^{18} \mathrm{~F}$ fluorothymidine in patients with gliomas. J Nucl Med. 2006;47:1612-1621.

10. Smith HW, Marshall CJ. Regulation of cell signalling by uPAR. Nat Rev Mol Cell Biol. 2010;11:23-36.

11. Solberg H, Ploug M, Hoyer-Hansen G, Nielsen BS, Lund LR. The murine receptor for urokinase-type plasminogen activator is primarily expressed in tissues actively undergoing remodeling. J Histochem Cytochem. 2001;49: 237-246.

12. Ploug M, Ostergaard S, Gardsvoll H, et al. Peptide-derived antagonists of the urokinase receptor: affinity maturation by combinatorial chemistry, identification of functional epitopes, and inhibitory effect on cancer cell intravasation. Biochemistry. 2001;40:12157-12168.

13. Li ZB, Niu G, Wang H, et al. Imaging of urokinase-type plasminogen activator receptor expression using a ${ }^{64} \mathrm{Cu}$-labeled linear peptide antagonist by microPET. Clin Cancer Res. 2008;14:4758-4766.

14. Persson M, Madsen J, Ostergaard S, Ploug M, Kjaer A. ${ }^{68}$ Ga-labeling and in vivo evaluation of a uPAR binding DOTA- and NODAGA-conjugated peptide for PET imaging of invasive cancers. Nucl Med Biol. 2012;39:560-569.

15. Persson M, Liu H, Madsen J, Cheng Z, Kjaer A. First ${ }^{18} \mathrm{~F}$-labeled ligand for PET imaging of uPAR: in vivo studies in human prostate cancer xenografts. $\mathrm{Nucl} \mathrm{Med}$ Biol. 2013;40:618-624.

16. Persson M, Madsen J, Jørgensen TJD, Jensen KJ, Kjaer A, Ploug M. Improved PET imaging of uPAR expression using new ${ }^{64} \mathrm{Cu}$-labeled cross-bridged peptide ligands: comparative in vitro and in vivo studies. Theranostics. 2013;3:618-632.

17. Persson M, Madsen J, Ostergaard S, et al. Quantitative PET of human urokinasetype plasminogen activator receptor with ${ }^{64} \mathrm{Cu}$-DOTA-AE105: implications for visualizing cancer invasion. J Nucl Med. 2012;53:138-145.

18. Persson M, Nedergaard MK, Brandt-Larsen M, et al. Urokinase-type plasminogen activator receptor as a potential PET biomarker in glioblastoma. J Nucl Med. 2016;57:272-278.

19. Lin L, Gardsvoll H, Huai Q, Huang M, Ploug M. Structure-based engineering of species selectivity in the interaction between urokinase and its receptor: implication for preclinical cancer therapy. J Biol Chem. 2010;285:10982-10992.

20. Persson M, Skovgaard D, Brandt-Larsen M. First-in-human uPAR PET: imaging of cancer aggressiveness. Theranostics. 2015;5:1303-1316.

21. Persson M, Rasmussen P, Madsen J, Ploug M, Kjaer A. New peptide receptor radionuclide therapy of invasive cancer cells: in vivo studies using ${ }^{177} \mathrm{Lu}-\mathrm{DOTA}-$ AE105 targeting uPAR in human colorectal cancer xenografts. Nucl Med Biol. 2012;39:962-969. 